\title{
Structure and dynamics of ion clusters in linear octupole traps: Phase diagrams, chirality, and melting mechanisms
}

\author{
E. Yurtsever* and E. D. Onal \\ Koç University, Rumelifeneriyolu, Sariyer, Istanbul TR-34450, Turkey \\ F. Calvo \\ LASIM, Université de Lyon and CNRS UMR 5579, 43 Bd du 11 Novembre 1918, FR-69622 Villeurbanne Cedex, France
}

(Received 15 March 2011; published 24 May 2011)

\begin{abstract}
The stable structures and melting dynamics of clusters of identical ions bound by linear octupole radiofrequency traps are theoretically investigated by global optimization methods and molecular dynamics simulations. By varying the cluster sizes in the range of 10-1000 ions and the extent of trap anisotropy by more than one order of magnitude, we find a broad variety of stable structures based on multiple rings at small sizes evolving into tubular geometries at large sizes. The binding energy of these clusters is well represented by two contributions arising from isotropic linear and octupolar traps. The structures generally exhibit strong size effects, and chiral arrangements spontaneously emerge in many crystals. Sufficiently large clusters form nested, coaxial tubes with different thermal stabilities. As in isotropic octupolar clusters, the inner tubes melt at temperatures that are lower than the overall melting point.
\end{abstract}

DOI: 10.1103/PhysRevA.83.053427

PACS number(s): 37.10.-x, 36.40.Ei, 52.27.Jt, 52.27.Gr

\section{INTRODUCTION}

Laser cooling of ion clouds confined in electromagnetic Paul or Penning traps has recently become efficient enough to produce Coulomb crystals, where large sets of charged particles arrange into various geometries that are observable by fluorescence. The very low temperature reached by the ions has led to various applications in fundamental physics [1,2], high-resolution spectroscopy and frequency standard [3,4], or information processing and quantum computing [5]. But the possibility of studying a many-body system bound by longrange forces also offers an interesting problem for statistical mechanics [6,7]. So far, most experiments on laser-cooled Coulomb crystals have been carried out on quadrupole traps, where confinement is harmonic (but not necessarily isotropic) in all directions [8-13]. Depending on the trap geometry, the ions initially arrange into linear strings along the main axis before exhibiting well-defined shells and cylindrical shapes of oblate or prolate character [13] and eventually the body-centered-cubic Wigner crystal structure [7].

In contrast with the quadrupole trap, where the density in the ion clouds is essentially uniform up to some shell deformations, higher-order confinements result in much weaker electric fields near the center, hence pushing the ions away in the corresponding directions [14]. The field-free central region is useful for the storage of neutral species that could react with the ions. When combined with buffer gas cooling techniques, this has led to interesting advances in cold ion-molecule collisions [15,16] but also ion clocks for space navigation [17]. The crystallization of ion clouds in linear rf octupole trap by laser-cooling techniques has been reported only recently by the Okada group $[18,19]$, who assisted their experiments with molecular dynamics simulations [18] for crystals containing

*eyurtsev@ku.edu.tr up to 250 ions. These authors found that the ions arrange into rings, then into cylinders as their numbers increase [18].

The linear rf octupole ion trap is an intermediate device where confinement is quadrupolar along the main axis but octupolar in the two remaining directions. This combination roughly explains the structural features found by Okada and co-workers based on the structures known theoretically for ion crystals in isotropic traps [6,7,20]. The dense versus hollow clouds predicted for the quadrupolar and octupolar traps, respectively, immediately suggest that the crystals in linear octupole traps should consist of a dense arrangement along a single axis with a hollow structure along the two other axes, which is nothing else but a cylinder. Another important difference between the quadrupolar and octupolar traps is the crystallization and melting mechanisms. Computer simulations have shown that, for clusters in harmonic traps, melting is initiated from the outer layers [21] in a way very similar to dense materials [22]. Coulomb crystals in octupolar traps, on the other hand, melt from their core [20]. Melting in ion clusters is also of interest in plasma physics, especially in the case of dusty plasmas that can form similar crystal structures, referred to as "dust balls" [23,24].

The results of Okada and co-workers $[18,19]$ and the current interest for Coulomb crystals in linear octupole traps [25] thus raise many questions: (i) How do the ions arrange in clusters containing more than a couple of hundred particles, and can trends be drawn for larger sizes? (ii) Can the influence of the trap anisotropy on the cluster shape be quantified? (iii) Are there cluster size effects [26] specific to these types of systems and interactions? (iv) What are the mechanisms of crystallization in linear octupolar traps, and (how) do they differ from the mechanisms found for isotropic confinements? Our goal in this paper is to address these issues by performing systematic global optimization to locate the stable structures of Coulomb crystals, as well as molecular dynamics simulations to get a detailed picture of the melting process, both being achieved under various trap conditions. 
Based on our calculations, we are able to locate important structural families into specific diagrams and to determine the relative roles of quadrupolar and octupolar confinements onto the melting mechanisms.

This paper is organized as follows. In the next section, we briefly describe the model and the introduction of a single-trap anisotropy parameter resulting from the use of reduced units. The numerical protocols for global optimization and molecular dynamics simulations are also detailed in this section. Section III presents and discusses our results on the stable structures, with some illustrative examples, an emphasis on their energetic stability and the possible magic numbers, together with some possible emergence of chirality in many clusters. Section IV discusses the finite-temperature dynamics of some large clusters and shows how some ion-resolved indicators can be used to identify the melting mechanisms. A summary and some concluding remarks are finally given in Sec. V.

\section{MODEL AND METHODS}

In this section, we start by briefly describing the physical model and its reduction to dimensionless units. We then give some details about the computational methods used for global structure optimization and dynamical trajectories.

\section{A. Potential energy surface}

We consider a set of $N$ identical ions carrying a single charge $q=+e$ each, with the position of ion $i$ being denoted as $\mathbf{r}_{i}=\left(x_{i}, y_{i}, z_{i}\right)$. The ions are confined statically along the linear axis $(z)$ and radially by a radiofrequency electromagnetic field created by eight electrodes parallel to $z$. The static potential $V_{s}(\mathbf{r})$ results from two opposite electrodes and satisfies the Laplace equation; its expression can be written as [18]

$$
V_{s}(\mathbf{r})=q A\left(z^{2}-\rho^{2} / 2\right),
$$

where, by definition, $\rho=\sqrt{x^{2}+y^{2}}$ will be referred to as the radial distance, and $A$ is a physical constant that depends on the geometric and voltage specifications of the trap. The octupole contribution to the confinement potential $V_{o}(\mathbf{r})$ will be simplified by omitting the time-dependent (radiofrequency) part, which amounts to neglecting the so-called residual micromotion. The resulting expression for $V_{o}$ can be shown as [15]

$$
V_{o}(\mathbf{r})=\frac{q^{2} B}{m} \rho^{6},
$$

where again $B$ is a physical constant depending on the trap and $m$ is the ion mass. Once we include the Coulomb repulsion $q^{2} / 4 \pi \varepsilon_{0} r_{i j}$ between ions $i$ and $j$ distant by $r_{i j}$, the total potential energy $E\left(\left\{\mathbf{r}_{i}\right\}\right)$ felt by the set of ions can be put into dimensionless units by using scaled variables $r \rightarrow \tilde{r}=\alpha_{r} r$ and $E \rightarrow \tilde{E}=\alpha_{E} E$. The scaling parameters $\alpha_{r}$ and $\alpha_{E}$ are then taken in such a way that the expression of $\tilde{E}$ simplifies. Due to the presence of two independent contributions to the trap potential, $\tilde{E}$ still contains one parameter and we have chosen to attribute it to the static potential as

$$
E\left(\left\{\mathbf{r}_{i}\right\}\right)=\sum_{i<j} \frac{1}{r_{i j}}+\sum_{i} \rho_{i}^{6}+\alpha \sum_{i}\left(z_{i}^{2}-\frac{\rho_{i}^{2}}{2}\right),
$$

where, for simplicity of notations, the tildes have been dropped from all variables. All information about the trap and ion characteristics is thus contained in the parameters $\alpha, \alpha_{r}$, and $\alpha_{E}$, their expressions being obtained from straightforward algebra:

$$
\begin{gathered}
\alpha=(A / q)(m / B)^{3 / 7}\left(4 \pi \varepsilon_{0}\right)^{4 / 7}, \\
\alpha_{r}=\left(m / 4 \pi \varepsilon_{0} B\right)^{1 / 7}, \\
\alpha_{E}=q^{2}(B / m)^{1 / 7}\left(4 \pi \varepsilon_{0}\right)^{-6 / 7} .
\end{gathered}
$$

The parameter $\alpha$ determines the strength of the static term relative to the octupole contribution. As will be seen below, it strongly affects the stable structures of the ion crystals and it will consequently be referred to as the trap anisotropy parameter. To determine the range of values relevant for realistic traps, we have simply taken appropriate values for $A$ and $B$ in the work by Okada and co-workers [18], and for $\mathrm{Ca}^{+}$ions $\alpha$ is approximately $\alpha=4.223$ reduced units. In that case, the unit distance and energy are given by $\alpha_{r}=0.624 \mathrm{~mm}$ and $\alpha_{E}=26.8 \mathrm{mK}$, respectively. After some preliminary exploration, we found a suitable range of $\alpha$ to be $1-10$, with occasional excursions below 1 or above 10 . The exploration we have carried out indicates that the 1-10 range gives rise to the most structural diversity for clusters containing less than 200 ions.

\section{B. Computational tools}

As in our earlier work on trapped Coulomb clusters [20,27-30], we used the basin-hopping algorithm [31] to locate global minima of the clusters. The basin-hopping strategy allows us to locate minima even though there may exist several funnels along the potential energy surface (PES) of the cluster. A Monte Carlo trajectory involving large amplitude motions is subjected to successive optimizations that sample the PES in an efficient manner. To improve the chances to successfully locate the global minima, several independent Monte Carlo trajectories starting from different initial random minima were used. Except for large clusters, less than 10 trajectories with 1000 optimizations were sufficient to locate global minima, a rather small number owing to the long range of the Coulomb potential [32].

The optimizations were carried out for all cluster sizes $4 \leqslant N \leqslant 200$ and $1 \leqslant \alpha \leqslant 10$ as well as for a few large clusters in the range $N=200-1000$. Once the global minima are found, we characterized them in terms of number of shells, global ellipsoid shape, and as functions of both size and trap anisotropy $\alpha$. We also attempted to correlate structural and energetic stabilities by computing second energy derivatives and a chirality index.

The finite-temperature behavior was addressed using classical molecular dynamics at constant energy. The velocity Verlet method was employed with a time step of $\delta t=10^{-3}$ reduced units, and the trajectories were propagated for $5 \times 10^{6}$ time steps following $10^{6}$ thermalization steps. The kinetic 
temperature extracted from these simulations, measured in reduced units equivalent to $1 / \Gamma$ from plasma physics, ranges from $10^{-2}$ to $10^{-1}$. At higher temperatures, the ions reach regions where the octupolar confinement increases, putting an upper limit on $\delta t$ compatible with total energy conservation. Note that, due to the anisotropic confinement, the angular momentum is not conserved except along the $z$ axis. After each trajectory, the final configuration served as initial condition for the next trajectory at higher energy.

\section{STABLE STRUCTURES}

\section{A. An overview}

The field of clusters and nanoparticles is largely marked by the existence of strong size-dependent physical properties [26,33]. The Coulomb clusters are not exceptions, and the geometrical characteristics of the clusters are strongly affected by the value of $\alpha$ in addition to the number of particles. As shown by Okada and co-workers [18], and confirmed below, the anisotropy of the potential of Eq. (3) results in cylindrical hollow structures except for a small number of particles. As in Coulomb clusters in dust plasmas, small clusters in ion traps are two dimensional and usually found in the form of single shells. For example, for $\alpha=4, N<9$ and $\alpha=10, N<13$, all clusters form regular planar, circular structures. As the size grows, the structures become three dimensional, cylindrical, and may include multiple shells.

To classify structures and understand the stability-structure relationship, we utilize two distinct features. Particles lying on the same $x y$ plane are called layers or rings, whereas particles that have very similar $\rho$ values are denoted as tubes. These layers and tubes can be found as perfectly regular structures or they may display a degree of disorder. These relatively irregular structures are not due to the incompleteness of the optimization but, rather, they are characteristic of the potential energy surface.

In Fig. 1, the top and side views of selected clusters with $\alpha=1$ are given. The small value of $\alpha$ allows particles to escape
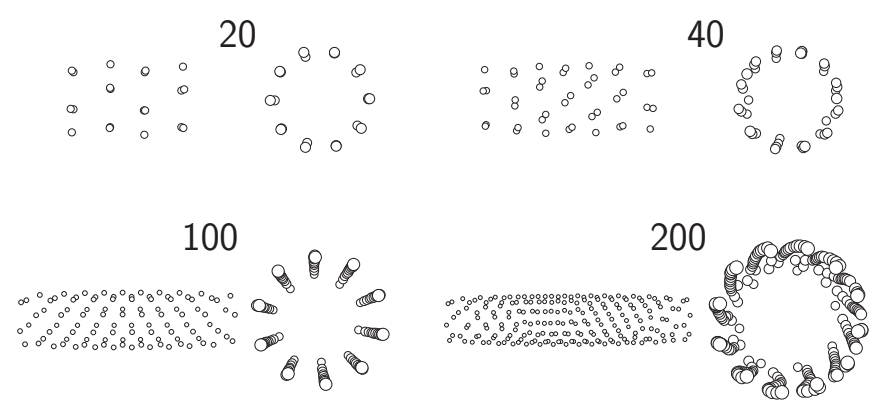

1000

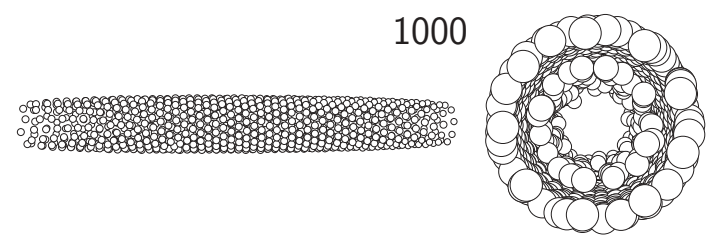

FIG. 1. Selected global minima found for ion clusters in the trap with $\alpha=1$. For each cluster, two views along and perpendicular to the trap axis are shown.
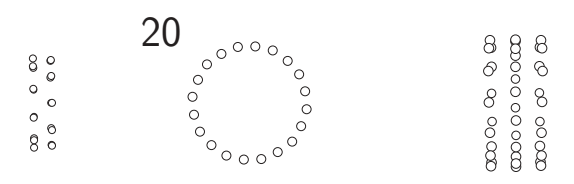

40
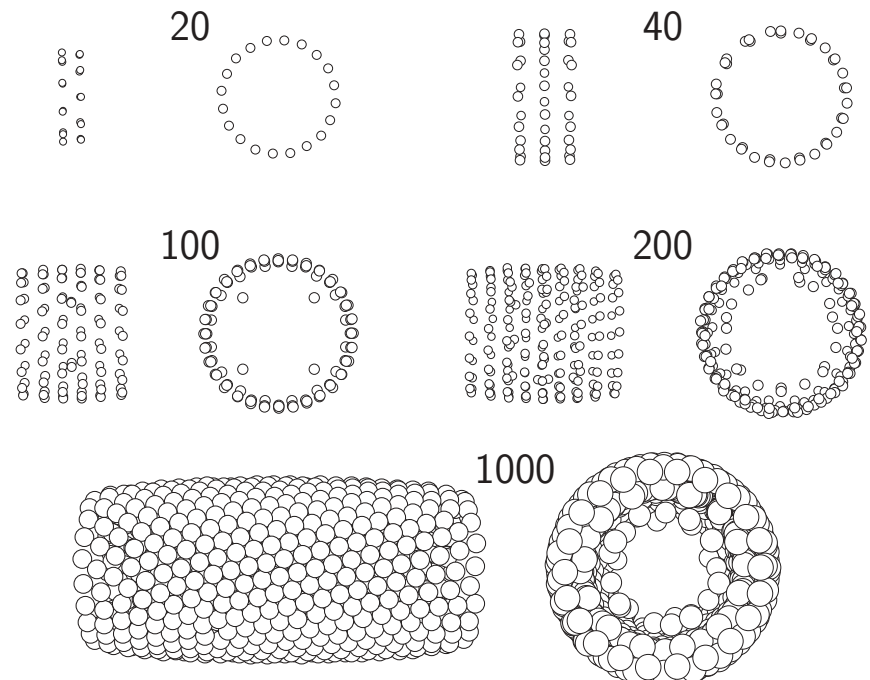

1000

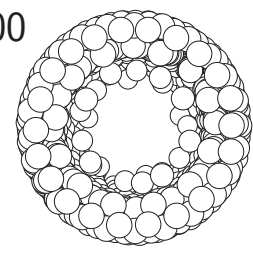

FIG. 2. Selected global minima found for ion clusters in the trap with $\alpha=10$. For each cluster, two views along and perpendicular to the trap axis are shown.

in the $z$ direction, hence resulting in elongated structures. For this form of potential, small clusters do not display perfectly aligned layers or tubes. Only for sizes $N>100$ are more ordered structures observed. These large clusters form hollow cylindrical structures with barrel-like shapes in which the radii at the top and the bottom portions are smaller compared to those around the middle. The particles at these extremities of the larger clusters are held together rather loosely since the potential is dominated by the large $z$ values. The well-defined tubes occur much more frequently than perfect layer-by-layer structures.

On the other hand, the clusters obtained for $\alpha=10$ show different shape characteristics (see Fig. 2). The effect of the $z^{2}$ contribution is now stronger compared to the radial potential and it results in more perfect layers and tubes. In addition, due to the stronger confining potential in the $z$ direction, the clusters are more compact.

\section{B. Structural diagrams}

For the range of $\alpha$ values studied here, almost all clusters with fewer than 100 ions form single-tube structures. Then, as new ions are added, new tubes start to grow. This growth is not monotonous such that, while increasing the size, the added ion may or may not go into the inner tube. In fact, in the majority of cases, the new ions are added to the outer shell. Only after a sufficiently large number of ions form the second shell (or the outer shell reaches a limit size) is a continuous addition to the inner shell observed. For the cases where only a small numbers of ions are in the inner shell, there is a certain degree of ambiguity in defining whether a cluster has one or more tubes.

In Fig. 3, we use these characteristics of the global minima to construct a structural phase diagram consisting of rings, single tube, or two or more tube regions as a function of both $N$ and $\alpha$. There is only a small regime where single planar rings are observed. As it is expected, more ions can form such stable structures when $\alpha$ increases. As $\alpha$ increases, the 

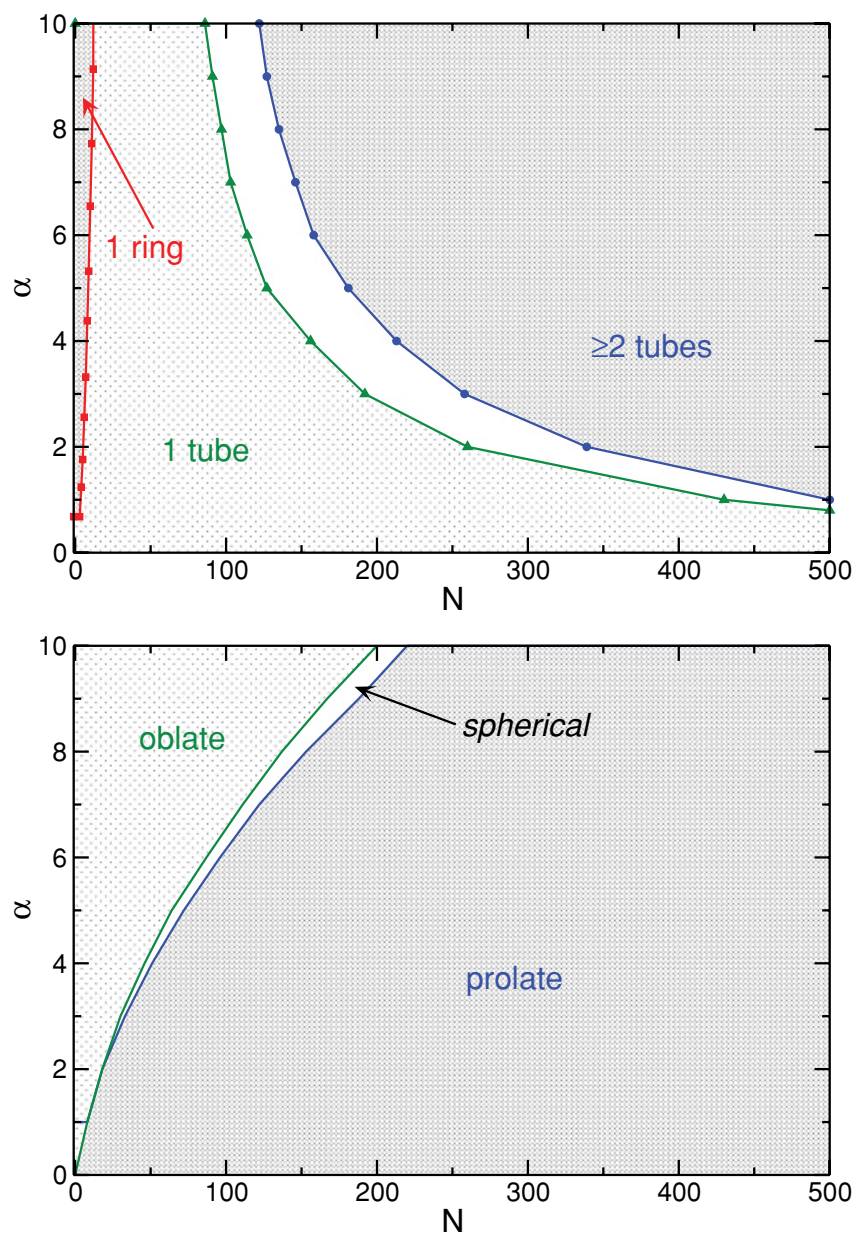

FIG. 3. (Color online) Structural diagrams for ion clusters in linear octupole rf traps, as a function of size $N$ and trap anisotropy $\alpha$. The upper panel shows the partitioning of the cluster into single-ring, single-tube, and multiple-tube arrangements, a second tube being recognized if the number of constituent atoms exceeds 5. The lower panel highlights the global cluster shape in terms of the main deformation parameter and the prolate or oblate character. The spherical shape is defined up to $2.5 \%$ deviation to asphericity.

transition regime where the global minimum changes from single tube to multiple tubes occurs at smaller clusters. For $\alpha<1$, even 500 particles form a single tube. On the other extreme, for $\alpha=10,500$ particles form multiple tubes, even though the radial distributions become blurred near the center in the same way as in our previous investigation on isotropic octupolar traps [20].

Another way of classifying the global minima is based on the shape analysis in terms of equivalent ellipsoids, or whether they should be considered as discoids (oblate) or elongated (prolate) configurations. The effect of $\alpha$ on the elongation of the cluster was mentioned above and can be further seen in Figs. 1 and 2. The deformation parameters are straightforwardly obtained from the principal momenta of inertia after diagonalizing the inertia tensor. In our case, we consider the shape to be an oblate ellipsoid if a single momentum exceeds the other two by more than $2.5 \%$, and a prolate ellipsoid if one momentum is lower than the other two by the same amount. Otherwise, the shape is considered to be globally spherical. The corresponding phase diagram, depicted in the lower panel of Fig. 3, is essentially dominated by prolate structures with a very narrow regime of almost spherical clusters. This domination reflects the mainly octupolar character of the trap, which compresses the ion crystals in two directions, leaving them to escape more easily along the remaining axis. In a hypothetical trap with a one-dimensional octupolar confinement, but a quadrupolar confinement in the two other directions, we would get exactly the opposite results, with mostly discotic shapes as the harmonic trap becomes stronger.

\section{Energetic stability}

Both experimental and theoretical studies of clusters have shown that their stability displays a strong dependence on size, which is usually well described by scaling laws in large systems but with nonmonotonic behavior in small systems [26,33]. The higher stabilities of specific systems result from geometric or electronic shell closure, and have been reported for different compounds such as noble gases and parahydrogen [34-36], water [37], metallic [38], or carbon [39] clusters, to name a few.

Before addressing the possible existence of such magicnumber clusters in the present linear octupole trap, we look for possible generic behavior that may arise from the general expression of the energy [Eq. (3)]. In isotropic traps, mean-field models $[20,40]$ predict that the potential energy $E(N)$ should scale with a specific power of size, namely, $E(N) \propto N^{5 / 3}$ in the quadrupolar case and $E(N) \propto N^{13 / 7}$ in the octupolar case. It is then tempting to fit the energies obtained for the linear octupole trap using an additive combination of these two expressions,

$$
E(N, \alpha) \approx A_{q}(\alpha) N^{5 / 3}+A_{o}(\alpha) N^{13 / 7},
$$

where the parameters $A_{q}$ and $A_{o}$ are related to the quadrupolar and octupolar confinements, respectively, and only depend on $\alpha$. In this fitting procedure, some of the small sizes were left out. This was due to the particular shape of the quadrupolar component of the potential in Eq. (3), which, due to the Laplace constraint, can be negative and not accurately represented by a simple $a N^{5 / 3}$ form. This behavior mostly occurs for large $\alpha$ and for cluster sizes below 10 ions. The cohesion energies obtained from global optimization are represented in Fig. 4 together with their fitted form based on Eq. (5) for the typical trap anisotropies of 1,4 , and 10 . The values of the parameters $A_{q}$ and $A_{o}$ are given in Table I for $\alpha$ in the entire range 1-10. As can be seen from Fig. 4, this empirical expression correctly accounts for the total potential energy of the clusters, at least in the range $1 \leqslant \alpha \leqslant 10$. The coefficients of the fits show regular variations, with increasingly negative value for the quadrupolar coefficient $A_{q}$ as $\alpha$ increases.

Special stabilities do not stand out from the fitted values, which suggests that additional parameters need to be estimated to locate possible magic numbers. Here, we use the rather customary derivatives of energy with respect to the particle number. The first derivative $\Delta E(N)=E(N+1)-E(N)$ turns out to be an essentially smoothly varying function of $N$ and can not be used to identify magic sizes either. Therefore, we have resorted to the second derivative $\Delta_{2} E(N)=E(N+$ 


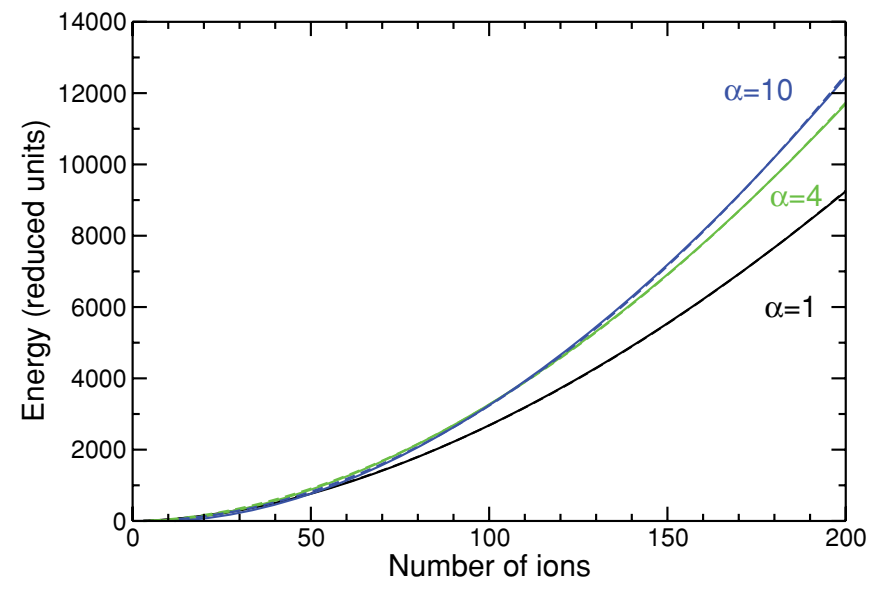

FIG. 4. (Color online) Cohesion energy as a function of cluster size for three trap parameters $\alpha=1,4$, and 10 . The raw data from global minimum structures are represented by solid lines, and the nearly superimposed dashed lines are fits based on the expression $E(N)=A_{q} N^{5 / 3}+A_{o} N^{13 / 7}$ (see text for details).

1) $+E(N-1)-2 E(N)$, which is much more sensitive to small changes in the energy. As in other properties, the existence and the nature of magic numbers depend on the static potential $\alpha$. In Fig. 5, we plot the discrete second derivative $\Delta_{2} E(N)$ for three values of $\alpha$. These three plots show the basic characteristics of the magic-size behavior of ion clusters in linear octupole traps. Even though the variations of $\Delta_{2} E$ with size are somewhat noisy, with no major peak or dip, they exhibit clearly different patterns as the trap parameter is varied. For the three values of $\alpha$ considered, multiple magic numbers can be identified from a high value of $\Delta_{2} E$ immediately surrounded by two low values for the neighboring sizes. These magic sizes occur in an alternating fashion for small $N$ values: the neighbors of a magic cluster are surrounded by two magic clusters. As the particle size increases, the appearance of magic clusters becomes less likely. There are no regular intervals between two adjacent magic sizes, and no particular periodicity can be noticed by plotting in logarithmic scale. Therefore, one can not correlate the highly stable forms just to the number of atoms; other structural considerations should be taken into account.

TABLE I. Fitting coefficients $A_{q}$ and $A_{o}$ for the cohesion energy of Coulomb crystals in the linear octupole trap as a function of the trap anisotropy $\alpha$.

\begin{tabular}{lcc}
\hline \hline$\alpha$ & $A_{q}$ & $A_{o}$ \\
\hline 1 & 0.4640 & 0.3245 \\
2 & 0.3613 & 0.4311 \\
3 & 0.2308 & 0.5168 \\
4 & 0.0896 & 0.5926 \\
5 & -0.0567 & 0.6623 \\
6 & -0.2065 & 0.7280 \\
7 & -0.3600 & 0.7913 \\
8 & -0.5165 & 0.8528 \\
9 & -0.6765 & 0.9135 \\
10 & -0.8393 & 0.9733 \\
\hline \hline
\end{tabular}

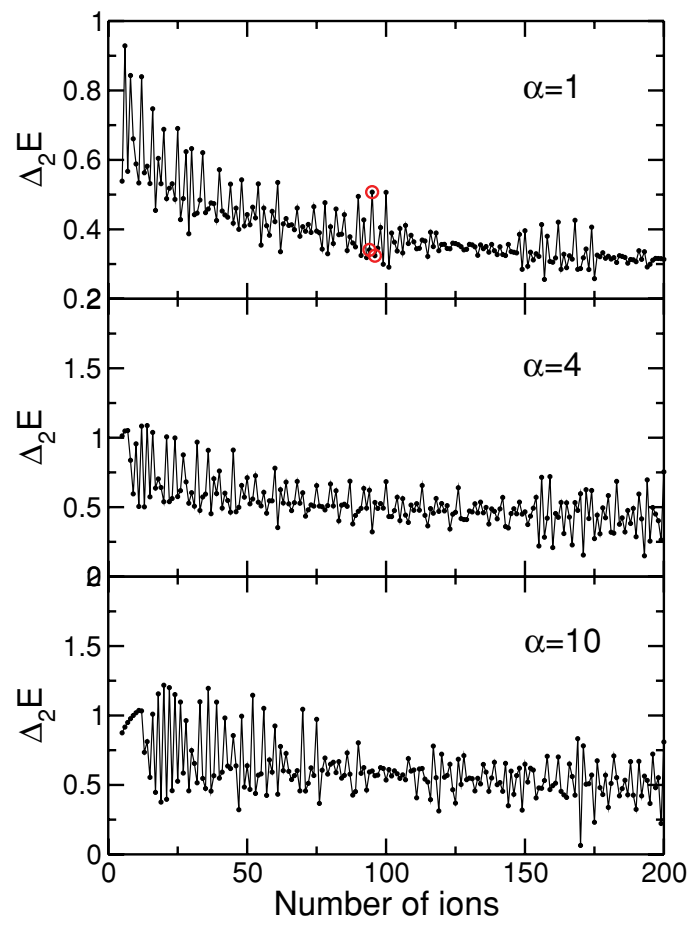

FIG. 5. (Color online) Second derivative of the cluster binding energy $\Delta_{2} E(n)=E(n+1)+E(n-1)-2 E(n)$ for the three trapping parameters characterized by $\alpha=1,4$, and 10 . The special stability near the 95 -ion crystal for $\alpha=1$ is highlighted. All energy derivatives are given in reduced units.

\section{Chirality}

The second energy derivatives show strong variations with size, indicating a significant dependence of cluster stability with the number of ions. In the size range $N \leqslant 200$, the magic numbers are generally associated with a perfect arrangement into identical, or at least commensurable, rings. The ring arrangement is conveniently visualized by plotting the distribution of axial distances $z$, and an example of such particularly stable structures is shown in Fig. 6, where the geometries found near size $N=95$ for the trap with $\alpha=1$ are also depicted. 95 ions are best located as a series of 19 rings of 5 atoms each, with a $2 \pi / 5$ rotation between adjacent rings in order to minimize Coulomb repulsion. This angle leads to an even-odd alternation between the rings in such a way that the surface structure is a triangular lattice equivalent to the two-dimensional Wigner crystal folded onto the tube. The corresponding distribution of $z$ distances displays 19 identical narrow peaks. The neighboring sizes both have low values of $\Delta_{2} E$, but rather different manifestations of their antimagic character. The crystal with 94 ions has a local defect under the form of a vacancy in one of the rings. This leads to important deformations that affect about 5 rings on both sides of the defective ring, with half of the 19 peaks in the $z$ distribution now being much broader.

On the other hand, the Coulomb crystal with 96 ions does not present any localized defect but a nearly continuous distribution of $z$ distances except at the two extremities. Rings are now much harder to assign in this structure, but direct visualization reveals a chiral pattern, with the ions being 
(a) $\mathrm{N}=94$

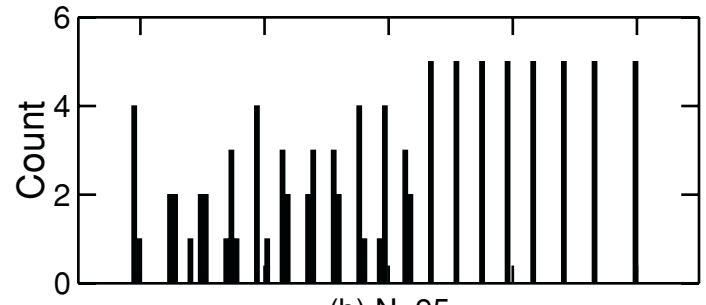

0.0000000000000
800000000000000
80000000

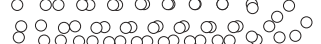

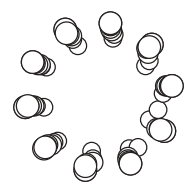

(b) $\mathrm{N}=95$
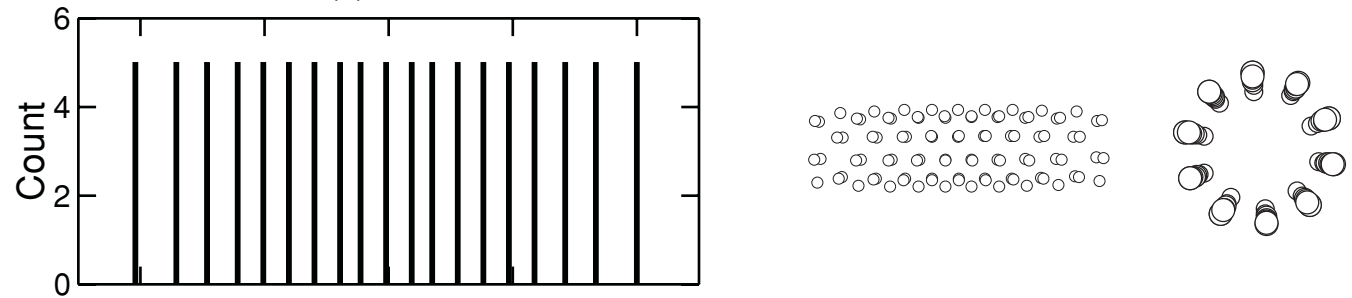

(c) $\mathrm{N}=96$
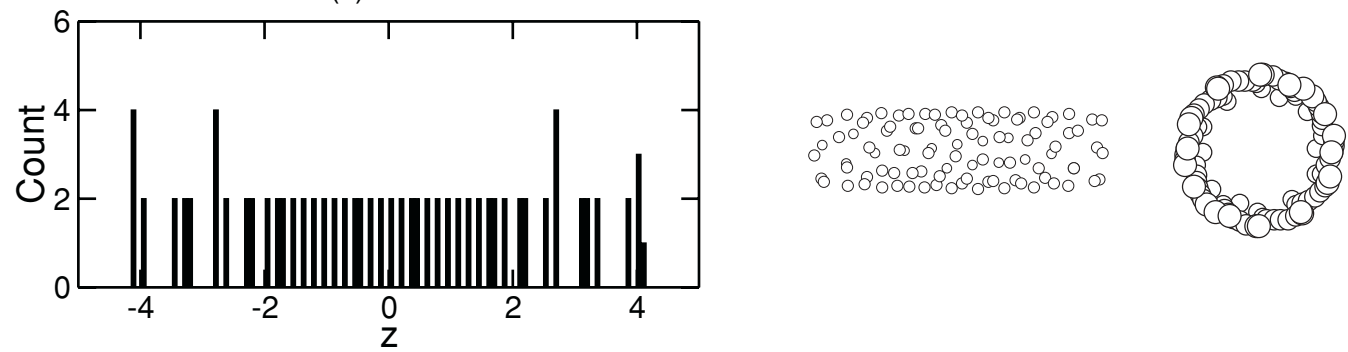

FIG. 6. Distribution of $z$ distances along the trap axis for cluster sizes near the magic number at $N=95$ and $\alpha=1$. The right panels illustrate the atomic structures of the clusters on their views along and perpendicular to the trap axis. Distances are measured in reduced units.

arranged in 10 spiraling strands. The spontaneous emergence of chirality in these clusters is caused by the difficulty of inserting the extra ion without disturbing the rings. Forming 16 perfect rings of 6 ions each appears not to be competitive, and a more stable arrangement is obtained by releasing the planar constraint ( $z=$ const.) on the rings, allowing some radial relaxation leading to comparable length and radius to the 95-ion cluster. Without this planar constraint, the rings undergo some rotation relative to the neighboring ring to minimize Coulomb repulsion, and the accumulation of this rotation gives the spiraling character. Interestingly, chiral patterns have also been reported in other atomic clusters such as $\mathrm{Au}_{n}$ [41], as well as in model building blocks for self-assembly [42]. Chiral tubes are also known to form in some proteins [43].

Several methods have been proposed in the literature to quantify chirality in molecular compounds [44-46]. In this paper, we have rationalized the extent of chirality in the Coulomb crystals by computing the following scaled parameter $\chi$ introduced by Solymosi and co-workers [47], and defined for the geometry $\mathbf{R}$ of a $N$-particle system by

$\chi(\mathbf{R})=\frac{1}{3 N^{3}} \sum_{i<j<k<\ell}\left(\mathbf{r}_{i j} \cdot \mathbf{r}_{j k}\right)\left(\mathbf{r}_{j k} \cdot \mathbf{r}_{k \ell}\right) \frac{\mathbf{r}_{i \ell} \cdot\left(\mathbf{r}_{i j} \times \mathbf{r}_{k \ell}\right)}{r_{i j}^{2} r_{j k}^{2} r_{k \ell}^{2} r_{i \ell}}$.

The chirality index exactly vanishes for an achiral structure, but has a nonzero (albeit small) value for any chiral arrangement. Contrary to other methods, it does not involve additional sampling of the orientational degrees of freedom of the structure and its mirror image. Unfortunately, the absolute magnitude of $\chi$ is difficult to interpret and even large chiral clusters such as helices may yield very low chirality indices depending on their spatial extension. Even though $\chi$ can hardly be used to compare two arbitrary structures on a quantitative footing, it is a valuable quantity to detect highly chiral or achiral geometries among a series. Figure 7 shows the variations of $\chi$ with size for the global minima of Coulomb
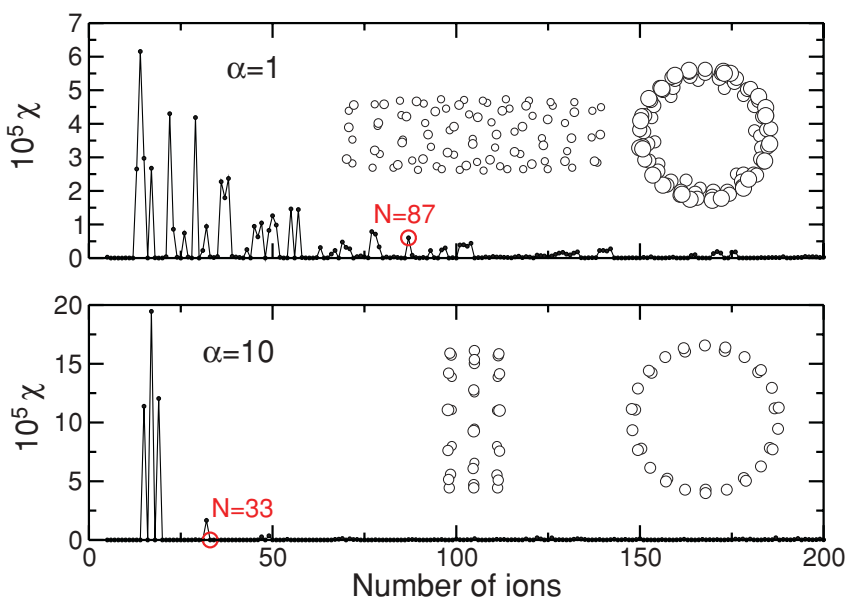

FIG. 7. (Color online) Scaled chirality index $\chi$ [see Eq. (6)] for all global minima of clusters in traps with $\alpha=1$ and 10 . Two clusters are highlighted (red circle, structure as an inset) for a highly chiral system at $\alpha=1$ and a highly symmetric system at $\alpha=10$. 
crystals in the range $N \leqslant 200$ and for trap characteristics of $\alpha=1$ and 10 . For each case, we highlighted two illustrative cluster structures.

The trap with smallest axial confinement $\alpha=1$ shows important variations in the chirality index of the Coulomb crystals with increasing size, with most global minima being chiral below 100 ions. The example chosen at size $N=87$ is a chiral structure without any clear ring arrangement and $\chi \sim 5 \times 10^{-6}$. The absence of any strand along the $z$ axis further indicates a nearly amorphous tube. For comparison, the clearly chiral but strandlike structure obtained for $N=200$ and $\alpha=1$ and represented in Fig. 1 has a much lower $\chi \simeq 10^{-7}$ that can not be noticed from Fig. 7 .

In the trap with $\alpha=10$, the ions arrange into single tubes only below 115 ions, after which an inner tube nucleates. Once the two tubes are formed, the spiral arrangements are hampered and the chirality index drops. However, $\chi$ is here very high in specific small size crystals consisting of defective ring structures, but several extremely low values of $\chi$ can be spotted in the range $N \leqslant 200$. The example of $N=33$, depicted as an inset in Fig. 7, is an achiral three-ring crystal with 11 ions in each ring and belonging to the unusual $D_{11 \mathrm{~h}}$ point group. Additional highly symmetric structures are found for $N=36\left(D_{12 h}\right), 75\left(D_{3 h}\right)$, or $108\left(S_{4}\right)$, for instance.

\section{FINITE-TEMPERATURE DYNAMICS}

Melting of ion crystals in quadrupole traps can be experimentally achieved by varying the trap parameters [48]. But, most related investigations in laser-cooled ion clouds and in dust-ball particles have relied on numerical experiments for the invaluable details they provide $[21,28,49]$. Using molecular dynamics simulations, Schiffer [21] has shown that, upon heating, a Coulomb crystal in a quadrupolar trap melts from the surface inward, a process also found in metallic nanoparticles [22]. In isotropic higher-order traps, mean-field models [14] predict that the hollow clusters should enlarge and broaden with increasing temperature; unfortunately, such approaches are not relevant for the melting problem itself. The simulations recently carried out by some of us were interpreted as a gradual melting initiated in the core and processing outward [20]. Because the structures formed by the ion clusters in the linear octupole trap show properties that are intermediate between those of clusters in the isotropic quadrupolar [6,7] and octupolar [20] traps, it is unclear how this mixed confinement will affect the melting scenario.

The melting process was monitored by performing molecular dynamics simulations and the order-to-disorder transition was probed by calculating the root-mean-square bond length fluctuation or Lindemann index $\delta$. Rather than discussing detailed size effects on a series of clusters, and along similar lines as our previous effort [20], we have chosen a single, rather large size of $N=1000$ to get insight into the general trends when the confinement parameter $\alpha$ is varied. At this size, the three crystals for $\alpha=1,4$, and 10 form at least two tubes, which is sufficient to investigate the core versus surface melting problem. Melting in a single-tube crystal was also simulated in order to question the possible importance of quadrupolar mechanisms, which could be manifested by the preliminary melting of the tube ends. $\alpha$ had to be decreased

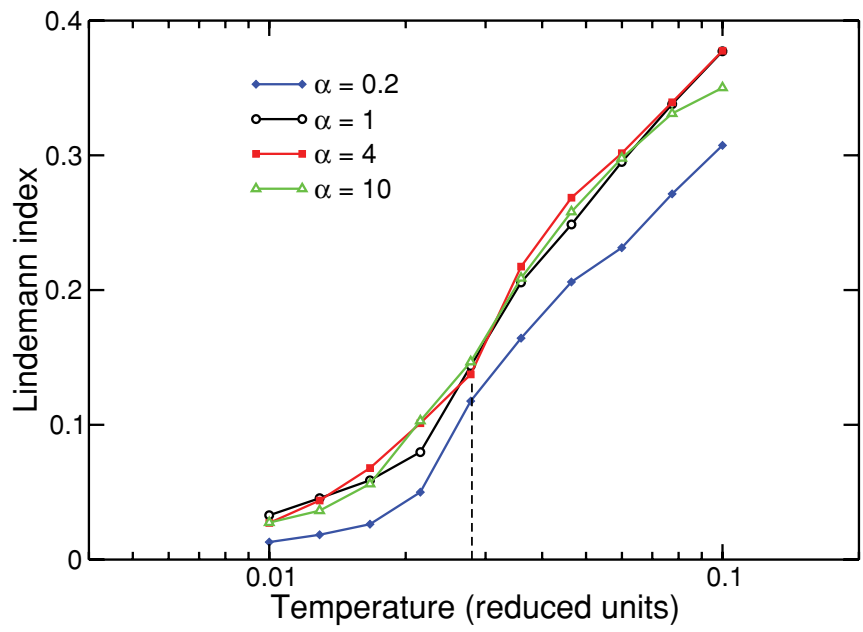

FIG. 8. (Color online) Particle-averaged Lindemann index against kinetic temperature, obtained from molecular dynamics simulations for 1000-ion clusters in various trap geometries $\alpha=0.2$, 1,4 , and 10 . The vertical dashed line highlights the overall melting temperature $T \simeq 0.028$ reduced units.

down to 0.2 for the putative global minimum to be made of a single tube.

The variations of the global Lindemann index for the four crystals are shown in Fig. 8 as a function of increasing reduced temperature. The Lindemann index varies smoothly for all clusters, but is always lower for the one-tube system with $\alpha=0.2$. Defining the melting point such that $\delta$ exceeds $10 \%-15 \%$ leads to the same value of $T \simeq 0.028$ reduced units, independent of $\alpha$. However, the variations of $\delta$ near this temperature are sharper for $\alpha=0.2$ and rather progressive for the other clusters, which suggests different mechanisms at the atomic level.

To unravel these detailed mechanisms at the resolution of single ions, we found it convenient to calculate the individual root-mean-square bond length fluctuation $\delta_{i}$ associated with each ion $i$,

$$
\delta_{i}=\frac{1}{N-1} \sum_{j \neq i} \frac{\sqrt{\left\langle r_{i j}^{2}\right\rangle-\left\langle r_{i j}\right\rangle^{2}}}{\left\langle r_{i j}\right.},
$$

in such a way that the global Lindemann index is simply the average of $\delta_{i}$ over all ions $\delta=\sum_{i} \delta_{i} / N$. To exploit the entire set of values $\left\{\delta_{i}\right\}$, we project the average position of each ion in cylindrical coordinates $\left(|z|, \rho=\sqrt{x^{2}+y^{2}}\right)$, taking the modulus of the axial distance for better visualization, and we associate a single circle with diameter $\delta_{i}$. Such projected plots are represented in Fig. 9 for the four clusters at the common temperature of $T=0.022$ reduced units, just before the global Lindemann index exhibits its most significant jump. These figures clearly show the multiple tube structures of the Coulomb clusters, with a single tube only for $\alpha=0.2$, and two tubes for the other clusters. For $\alpha=10$, inspection of the most stable structure reveals a third inner tube but, at the temperature considered here, this tube has lost its integrity. More interestingly, there is a strong correlation between the rigidity of the individual ions and their tube of origin. In all cases, the outermost tube consists of solidlike 

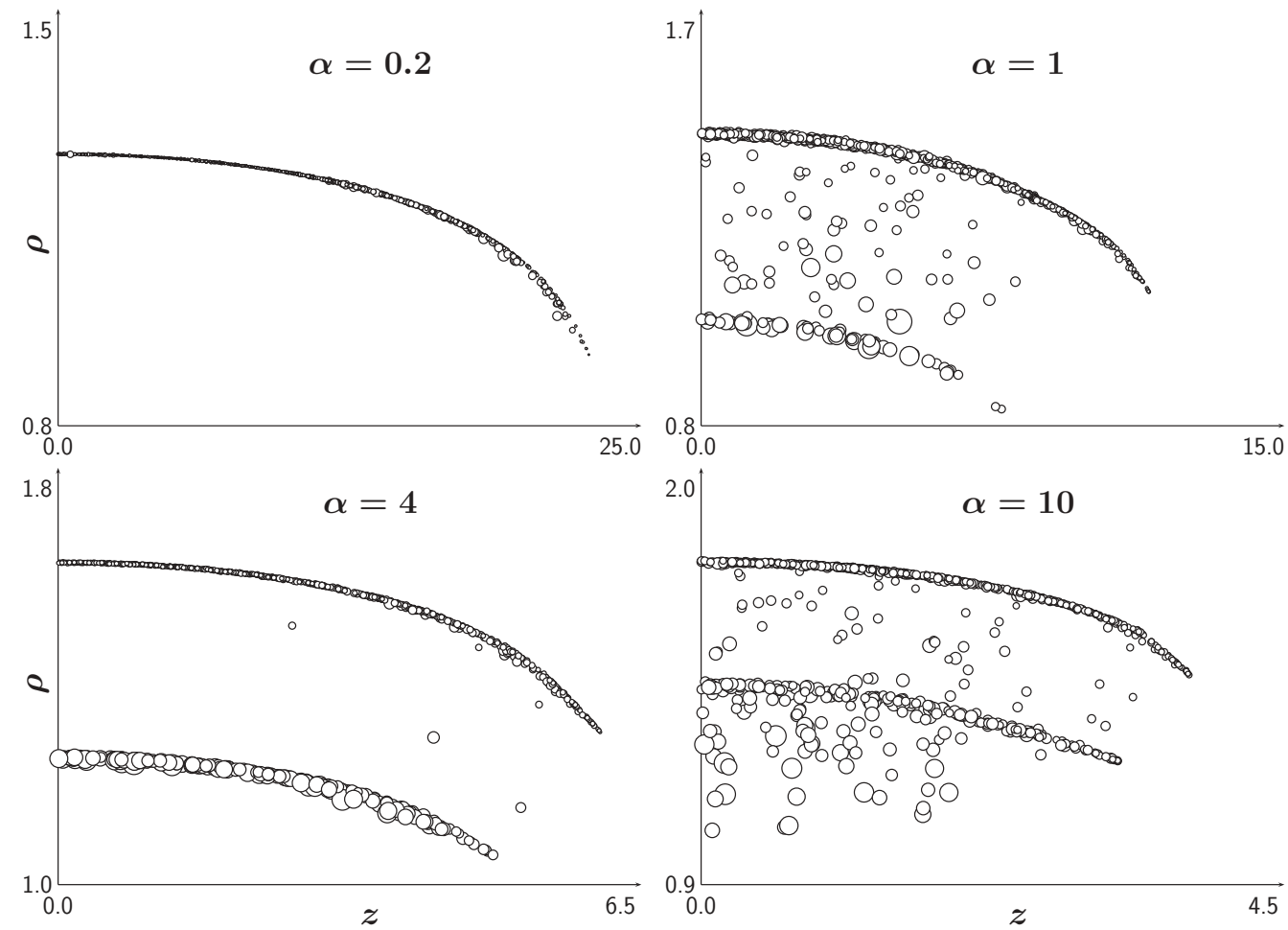

FIG. 9. Projections of cylindrical coordinates $(|z|, \rho)$ averaged along molecular dynamics trajectories at $T=0.022$ reduced units for 1000 -ion clusters in various trap geometries $\alpha=0.2,1,4$, and 10 . Each ion is depicted as a circle with diameter equal to its individual Lindemann index. All coordinates are measured in reduced units.

ions that essentially vibrate around their equilibrium positions. However, the interior tubes are much more disordered and can exhibit some phase separation with the outer rigid tube $(\alpha=4)$. As temperature increases, the ions are more and more likely to cross the space between the tubes, and this space becomes eventually filled with a low-density fluid that is especially visible for $\alpha=1$ and 10 . The clusters with $\alpha \geqslant 1$ thus display a typical core-melted state similar to the one previously found in the isotropic octupolar trap [20].

In the single-tube cluster with $\alpha=1$, the extremities show slightly higher mobilities than the central parts, which is the expected behavior for the purely quadrupolar trap [21]. This is not seen for the three other clusters, but often appears in smaller clusters under the form of the preliminary rotation of the two ends of a single tube, due to the presence of incommensurate rings. Apart from those rare cases, the present simulations show that the melting process is mainly driven by the octupolar confinement, unless the clusters only consist of a single tube.

Even though the four clusters considered in this paper exhibit different progressions in their melting process, it is remarkable that their global rigidities, as measured by the collective Lindemann index $\delta$, are rather similar, with a common effective melting point close to $T_{m} \simeq 0.028$ reduced units. This is interpreted as originating from two competing factors. As the axial confinement increases with $\alpha$, the ions in the outermost layer are also more strongly bound, which tends to delay the melting point. On the other hand, as internal tubes appear, they are much less confined in a weaker field region, hence their anticipated melting. The combination of these two effects balances the overall rigidity quantified by the averaged Lindemann index.

\section{CONCLUSIONS}

Despite simple pairwise interactions, clusters of identical ions in electromagnetic traps can exhibit a rich variety of structures and dynamics depending on the shape and anisotropy of the confinement. In this paper, we have investigated the stable configurations and melting behavior of some Coulomb clusters in realistic linear octupole traps, where confinement is harmonic along the trap axis. The global minima share the cylindrical symmetry of the confinement potential. In a broad range of anisotropies, they are first made of planar rings; then, as the number of ions increases, multiple rings are formed, evolving into a tube and eventually multiple tubes. The cohesion energies of the crystals are well represented by two additive contributions originating from equivalent isotropic confinements of the quadrupolar $\left(\propto N^{5 / 3}\right)$ and octupolar $\left(\propto N^{13 / 7}\right)$ types, but many magic-number clusters are found from the variations with size of the second energy derivative $\Delta_{2} E$. Among the nonmagic structures, chiral geometries are often seen, and the extent of chirality was quantified and found especially high in the one-tube clusters at small trap anisotropies. Finally, the melting dynamics was investigated by molecular dynamics trajectories in large representative clusters containing 1000 ions in various traps. Although the clusters do not show important variations in their melting point in a broad range of anisotropies, the mechanisms revealed by the atomresolved Lindemann indices involve some multistage melting 
affecting the inner tubes first, and progressing outward. At very small trap anisotropies $\alpha$, the extremities of single-tube clusters display lower rigidities than the center.

It thus appears that both the geometrical arrangement of ion clusters in linear octupole traps and their thermal stability result from a competition between the quadrupolar and octupolar contributions to the confinement potential. Another remarkable outcome of this study is the confirmation that octupolar confinement supports solid-liquid phase coexistence, a rather unusual feature in clusters [50]. It would be very interesting to further characterize this particular state of matter, for instance, by computing additional quantities such as diffusion constants or the rate at which particles thermalize either within a tube or in-between tubes.

Another aspect worth investigating would be the nonlinear dynamics of such Coulomb crystals. Previous efforts by several groups have focused on the quadrupole trap [27,51,52]. In particular, it was shown that the chaotic dynamics of the clusters undergoes two changes as temperature is increased, from a regular regime in the very cold, solidlike limit to a highly chaotic regime as the melting point is approached, and eventually to another regular regime at very high temperatures where the Coulomb interactions become negligible against the harmonic trap potential [51]. The octupole trap is not expected to give rise to regular dynamics in the latter gaseous state, which seems confirmed by preliminary calculations [53], but would require dedicated work also in the case of linear octupole confinement.

A landscape perspective, in which the important transitions between connected minima are obtained and the rates evaluated, would also be insightful, especially in relation with chirality. Other extensions of this paper could involve mixed clusters [54], include the natural micromotion arising from the radiofrequency traps [55], or try to determine whether scaling laws exist for the melting points as a function of size and trap anisotropy [20,21].

\section{ACKNOWLEDGMENT}

E.Y. would like to thank the Turkish Academy of Sciences for the support, and F.C. acknowledges the contribution of Xuan $\mathrm{Hu}$ on preliminary efforts. F.C. would also like to thank the Pôle Scientifique de Modélisation Numérique for a generous allocation of computer resources.
[1] S. Bize et al., Phys. Rev. Lett. 90, 150802 (2003).

[2] T. M. Fortier et al., Phys. Rev. Lett. 98, 070801 (2007).

[3] T. Rosenband et al., Science 319, 1808 (2008).

[4] M. Chwalla et al., Phys. Rev. Lett. 102, 023002 (2009).

[5] D. Kielpinski, B. E. King, C. J. Myatt, C. A. Sackett, Q. A. Turchette, W. M. Itano, C. Monroe, D. J. Wineland, and W. H. Zurek, Phys. Rev. A 61, 032310 (2000).

[6] R. Rafac, J. P. Schiffer, J. S. Handst, D. H. Dubin, and D. J. Wales, Proc. Natl. Acad. Sci. USA 88, 483 (1991).

[7] H. Totsuji, T. Kishimoto, C. Totsuji, and K. Tsuruta, Phys. Rev. Lett. 88, 125002 (2002).

[8] F. Diedrich, E. Peik, J. M. Chen, W. Quint, and H. Walther, Phys. Rev. Lett. 59, 2931 (1987).

[9] S. L. Gilbert, J. J. Bollinger, and D. J. Wineland, Phys. Rev. Lett. 60, 2022 (1988).

[10] J. D. Prestage, A. Williams, L. Maleki, M. J. Djomehri, and E. Harabetian, Phys. Rev. Lett. 66, 2964 (1991).

[11] W. M. Itano, J. J. Bollinger, J. N. Tan, B. Jelenković, X.-P. Huang, and J. D. Wineland, Science 279, 686 (1998).

[12] M. Drewsen, C. Brodersen, L. Hornekær, J. S. Hangst and J. P. Schiffer, Phys. Rev. Lett. 81, 2878 (1998).

[13] A. Mortensen, E. Nielsen, T. Matthey, and M. Drewsen, Phys. Rev. Lett. 96, 103001 (2006).

[14] J. Walz, I. Siemers, M. Schubert, W. Neuhauser, R. Blatt, and E. Teloy, Phys. Rev. A 50, 4122 (1994).

[15] D. Gerlich, Adv. Chem. Phys. 82, 1 (1992).

[16] R. Otto, J. Mikosch, S. Trippel, M. Weidemüller, and R. Wester, Phys. Rev. Lett. 101, 063201 (2008).

[17] L. Maleki and J. Prestage, Lect. Notes Phys. 648, 331 (2004).

[18] K. Okada, K. Yasuda, T. Takayanagi, M. Wada, H. A. Schuessler, and S. Ohtani, Phys. Rev. A 75, 033409 (2007).

[19] K. Okada, T. Takayanagi, M. Wada, S. Ohtani, and H. A. Schuessler, Phys. Rev. A 80, 043405 (2009).
[20] F. Calvo, C. Champenois, and E. Yurtsever, Phys. Rev. A 80, 063401 (2009).

[21] J. P. Schiffer, Phys. Rev. Lett. 88, 205003 (2002).

[22] P. Buffat and J. P. Borel, Phys. Rev. A 13, 2287 (1976).

[23] O. Arp, D. Block, A. Piel, and A. Melzer, Phys. Rev. Lett. 93, 165004 (2004).

[24] M. Bonitz, D. Block, O. Arp, V. Golubnychiy, H. Baumgartner, P. Ludwig, A. Piel, and A. Filinov, Phys. Rev. Lett. 96, 075001 (2006).

[25] C. Champenois, M. Marciante, J. Pedregosa-Gutierrez, M. Houssin, M. Knoop, and M. Kajita, Phys. Rev. A 81, 043410 (2010).

[26] J. Jortner, Z. Phys. D: At. Mol. Clusters 24, 247 (1992).

[27] E. Yurtsever, F. Calvo, and D. J. Wales, Phys. Rev. E 72, 26110 (2005).

[28] F. Calvo and E. Yurtsever, Eur. Phys. J. D 44, 81 (2007).

[29] E. Yurtsever and F. Calvo, Phys. Scr. 76, C111 (2007).

[30] E. Yurtsever and F. Calvo, Mol. Phys. 106, 289 (2008).

[31] D. J. Wales and J. P. K. Doye, J. Phys. Chem. A 101, 5111 (1997).

[32] P. A. Braier, R. S. Berry, and D. J. Wales, J. Chem. Phys. 93, 8745 (1990).

[33] R. L. Johnston, Atomic and Molecular Clusters (Taylor and Francis, London, 2002).

[34] O. Echt, K. Sattler, and E. Recknagel, Phys. Rev. Lett. 47, 1121 (1981).

[35] R. Brühl, R. Guardiola, A. Kalinin, O. Kornilov, J. Navarro, T. Savas, and J. P. Toennies, Phys. Rev. Lett. 92, 185301 (2004).

[36] S. A. Khairallah, M. B. Sevryuk, D. M. Ceperley, and J. P. Toennies, Phys. Rev. Lett. 98, 183401 (2007).

[37] H. M. Lee, S. B. Suh, P. Tarakeshwar, and K. S. Kim, J. Chem. Phys. 122, 44309 (2005). 
[38] W. D. Knight, K. Clemenger, W. A. de Heer, W. A. Saunders, M. Y. Chou, and M. L. Cohen, Phys. Rev. Lett. 52, 2141 (1984).

[39] H. W. Kroto, J. R. Heath, S. C. O'Brien, R. F. Curl, and R. E. Smalley, Nature (London) 318, 162 (1985).

[40] R. W. Hasse and V. V. Avilov, Phys. Rev. A 44, 4506 (1991).

[41] T. G. Schaaf and R. L. Whetten, J. Phys. Chem. B 104, 2630 (2000).

[42] S. N. Fejer, D. Chakrabarti, and D. J. Wales, ACS Nano 4, 219 (2010).

[43] S. Li, C. P. Hill, W. I. Sundquist, and J. T. Finch, Nature (London) 407, 409 (2000).

[44] A. B. Buda and K. Mislow, J. Am. Chem. Soc. 114, 6006 (1992).

[45] N. Weinberg and K. Mislow, J. Math. Chem. 17, 35 (1995).

[46] A. B. Harris, R. D. Kamien, and T. C. Lubensky, Rev. Mod. Phys. 71, 1745 (1999).
[47] M. Solymosi, R. J. Low, M. Grayson, and M. P. Neal, J. Chem. Phys. 116, 9875 (2002).

[48] L. Hornekær, N. Kjærgaard, A. M. Thommesen, and M. Drewsen, Phys. Rev. Lett. 86, 1994 (2001).

[49] H. Kählert and M. Bonitz, Phys. Rev. Lett. 104, 015001 (2010).

[50] D. J. Wales and J. P. K. Doye, J. Chem. Phys. 103, 3061 (1995).

[51] P. Gaspard, Phys. Rev. E 68, 056209 (2003).

[52] C. Antonopoulos, V. Basios, and T. Bountis, Phys. Rev. E 81, 016211 (2010).

[53] F. Calvo and E. Yurtsever, AIP Conf. Proc. 1197, 174 (2009).

[54] T. Matthey, J. P. Hansen, and M. Drewsen, Phys. Rev. Lett. 91, 165001 (2003).

[55] R. Blümel, C. Kappler, W. Quint, and H. Walther, Phys. Rev. A 40, 808 (1989). 